\title{
Low Loss, Tightly Coilable, Hollow Core Photonic Bandgap Fibers for Mid-IR Applications
}

\author{
N. V. Wheeler, M. N. Petrovich, A. M. Heidt, N. K. Baddela, E. N. Fokoua, J. R. Hayes,S. R. Sandoghchi, \\ F. Poletti and D. J. Richardson \\ Optoelectronics Research Centre, University of Southampton, Highfield, Southampton, SO17 1BJ, UK \\ e-mail: nvw1v10@orc.soton.ac.uk
}

\begin{abstract}
We describe low loss $(50 \mathrm{~dB} / \mathrm{km}$ at $3.3 \mu \mathrm{m})$ and low bend sensitivity mid-IR hollow core-photonic bandgap fiber. Gas sensing applications are highlighted by a methane spectrum recorded in our fiber.
\end{abstract}

Keywords-Hollow core-photonic bandgap fiber;mid-infrared; gas sensing;spectroscopy.

\section{INTRODUCTION}

Advances in optical sources such as quantum cascade lasers, frequency combs and broadband supercontinua operating in the mid-infrared (mid-IR) spectral region $(\sim 2-$ $20 \mu \mathrm{m})$ are driving an increasing interest in these wavelengths [1], [2]. The atmospheric transmission window between 3 and $5 \mu \mathrm{m}$ which overlaps with many fundamental absorption bands of molecular species opens up applications in fields such as environmental and industrial sensing, security and medical diagnostics. For these applications, a low loss optical fiber is highly beneficial; providing access to remote or hazardous areas and the means to fabricate compact, lightweight and portable devices.

Conventional, all-silica solid fibers, while integral to many applications at near-IR wavelengths, are not suited for mid-IR transmission due to the high material absorption of silica beyond $2 \mu \mathrm{m}$ [3]. Fibers fabricated from materials such as ZBLAN can provide low attenuation [4]; however, they cannot currently provide the combination of durability, bend insensitivity, modal quality and power handling offered by silica fibers in the near-IR. Hollow core photonic crystal fibers (HC-PCFs), consisting of an air core surrounded by a periodic array of air holes defined by a silica matrix, provide an alternative approach, capitalizing on the outstanding properties of silica glass in fiber fabrication while keeping attenuation low due to minimal overlap between the guided optical modes and the silica structure. Recent outstanding results [5], [6] have produced low attenuation fibers in

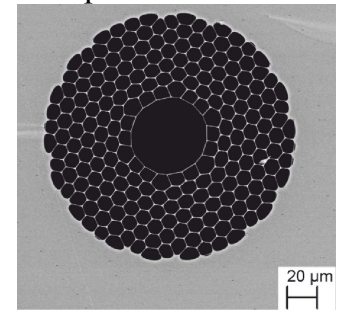

Fig. 1: SEM of an example mid-IR HC-PBGF remarkably simple fiber geometries.

In this paper we review our recent progress in fabrication of hollow core-photonic bandgap fiber (HC-PBGF, a type of HC-PCF) for mid-IR operation which combines low loss (50 $\mathrm{dB} / \mathrm{km}$ ) with low bend sensitivity [7] and is highly suited for applications in the 3 to $4 \mu \mathrm{m}$ spectral region such as high power laser delivery as well as gas sensing; this is highlighted by a high resolution spectrum recorded using $1.26 \mathrm{~m}$ of methane-filled HC-PBGF.

\section{FIBER FABRICATION}

The HC-PBGFs have a 19 cell core and were fabricated using the conventional two-step stack and draw process. A thin-walled core surround combined with careful pressure control during the fiber draw enables optimization of the final fiber geometry towards minimizing attenuation and maximizing the fiber's operating bandwidth. A scanning electron microscope image of an example fiber is shown in Fig. 1; the average core diameter is $50 \mu \mathrm{m}$, the average holeto-hole distance is $9.3 \mu \mathrm{m}$ and the relative hole size is 0.965 .

\section{FIBRE CHARACTERISATION}

The mid-IR transmission was measured using a custom built supercontinuum source which extends from 0.75 to 4 $\mu \mathrm{m}$ by using a diode seeded picosecond thulium-doped fiber amplifier operating at $2 \mu \mathrm{m}$ to pump a ZBLAN fiber. For signal detection a monochromator with $5 \mathrm{~nm}$ nominal resolution was initially used as it provided the means to detect transmission across the full fiber bandwidth. The fiber attenuation was calculated by a cutback loss measurement. Fig. 2a shows the cutback measurement for the fiber in Fig.1 which indicates a minimum loss of $130 \pm 50 \mathrm{~dB} / \mathrm{km}$ and sub$\mathrm{dB} / \mathrm{m}$ transmission from $3.1 \mu \mathrm{m}$ to beyond $3.6 \mu \mathrm{m}$. This loss result improves on the previous best HC-PBGF operating in this spectral region by an order of magnitude [8]. In the low loss transmission region of Fig. 2a several loss peaks are visible which can be attributed to $\mathrm{HCl}$ absorption; $\mathrm{HCl}$ is in the fiber due to the presence of chlorine in the dehydrated synthetic silica used as the raw fabrication material. These absorption lines are shown in more detail in Fig. 2b where a high resolution $(0.2 \mathrm{~nm})$ optical spectrum analyzer was used for detection. The minimum recorded loss was likely to be limited due to the presence of this species and the limited monochromator resolution, therefore the $\mathrm{HCl}$ was removed 

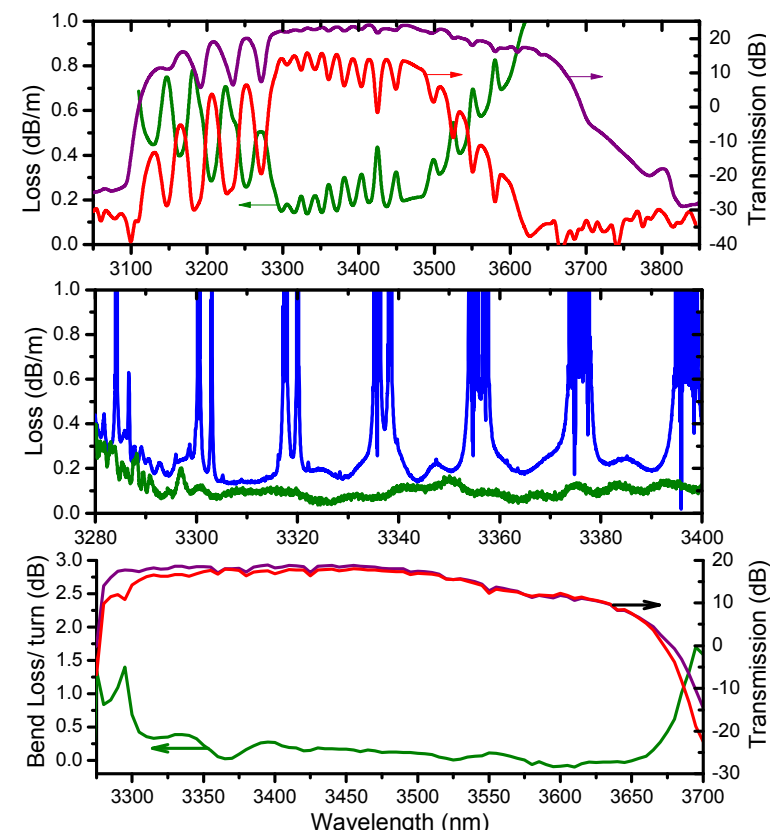

Fig. 2: (a) Cutback loss measurement (green line). Fiber lengths were 58 and $5 \mathrm{~m}$, shown by red and purple lines respectively, (b) Cutback loss measurements recorded with $0.2 \mathrm{~nm}$ resolution before and after purging fiber to remove $\mathrm{HCl}$ (blue and green lines respectively), (c) Bend loss per $5 \mathrm{~cm}$ turn (blue line) calculated from transmission of $5 \mathrm{~m}$ of HCPBGF with five coils of $5 \mathrm{~cm}$ diameter in the fiber (red line) and with the fiber loosely coiled (purple line).

by purging the fiber with Argon gas. The cutback measurement was then repeated (also shown in Fig. $2 b$ and at $0.2 \mathrm{~nm}$ resolution) and the minimum loss was found to be 50 $\mathrm{dB} / \mathrm{km}$. From extensive modelling of the fiber, further loss reduction and the ability to push the transmission window further into the mid-IR is expected through increasing the core diameter, e.g., by using a 37 cell HC-PBGF design [9].

The bend loss was found by comparing the transmission of $5 \mathrm{~m}$ of loosely coiled fiber, with the same fiber spooled to include five coils with $5 \mathrm{~cm}$ diameter. Fig. 2c shows that the resultant bend loss per turn is $<0.25 \mathrm{~dB} /$ turn over a $300 \mathrm{~nm}$ bandwidth; this is significantly lower than that reported recently for anti-resonant HC-PCFs designed for operation in the mid-IR showing that this fiber is suitable for applications requiring a compact device footprint.

\section{Application: Gas SENSING}

Hollow core fibers are particularly interesting for gas sensing as the fiber can act as the host for the gas species under investigation as well as the optical waveguide. Use of fundamental molecular absorption bands present in the midIR increases the potential sensitivity of a gas sensing device by orders of magnitude compared to the use of weaker, nearIR absorption bands. As an example of the sensitivity achievable using gas-filled HC-PBGF we filled $1.26 \mathrm{~m}$ of a fiber with a similar design to that in Fig. 1 with $1000 \mathrm{ppm}$ of methane in nitrogen. The transmission spectrum, shown in the lower part of Fig. 3, is in excellent agreement with data from HITRAN (shown in the top part of the figure). By using longer fibers in combination with more sophisticated

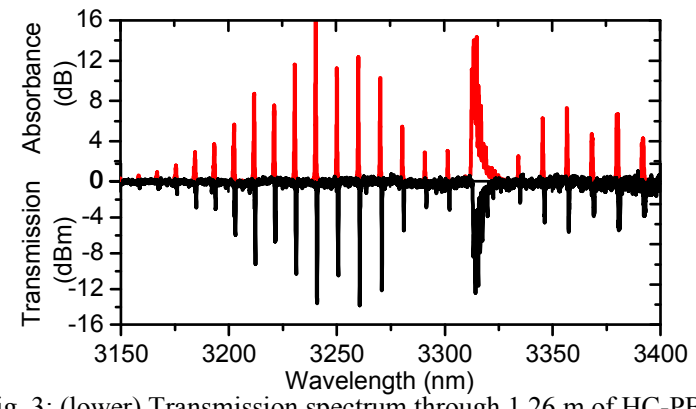

Fig. 3: (lower) Transmission spectrum through $1.26 \mathrm{~m}$ of HC-PBGF filled with $1000 \mathrm{ppm} \mathrm{CH}_{4}$ in $\mathrm{N}_{2}$, (top) theoretical transmission of

filled fiber using data from the HITRAN database [10].

detection techniques we envisage that sensitivity levels of the order of ppb will be easily achievable in these fibers.

\section{CONCLUSIONS}

We report fabrication of low loss $(50 \mathrm{~dB} / \mathrm{km})$ and low bend sensitivity HC-PBGFs for operation in the mid-IR. Potential applications of these fibers include gas sensing and high power laser delivery. We anticipate that further loss reduction can be achieved through optimization of the core geometry, in particular by increasing the core diameter to minimize modal overlap with the silica fiber framework.

\section{ACKNOWLEDGMENTS}

This work was supported by the UK EPSRC EP/H02607X/1. A.M.H. acknowledges funding from the EU FP7 Marie Curie Actions (grant 300859 (ADMIRATION)). F.P. gratefully acknowledges support from the Royal Society.

\section{REFERENCES}

[1] Y. Yao, A. J. Hoffman, and C. F. Gmachl, "Mid-infrared quantum cascade lasers," Nat. Photonics, vol. 6, no. June, pp. 432-439, 2012.

[2] A. Schliesser, N. Picqué, and T. W. Hänsch, "Mid-infrared frequency combs," Nat. Photonics, vol. 6, no. June, pp. 440-449, 2012.

[3] O. Humbach, H. Fabian, U. Grzesik, U. Haken, and W. Heitmann, "Analysis of $\mathrm{OH}$ absorption bands in synthetic silica," J. Non. Cryst. Solids, vol. 203, pp. 19-26, 1996.

[4] J. A. Harrington, Infrared Fibers and Their Applications. Bellingham, Washington USA: Society of Photo-Optical Instrumentation Engineers ., 2004.

[5] F. Yu, W. J. Wadsworth, and J. C. Knight, "Low loss silica hollow core fibers for 3-4 $\mu \mathrm{m}$ spectral region.," Opt. Express, vol. 20, no. 10, pp. 11153-8, May 2012.

[6] A. N. Kolyadin et al. "Light transmission in negative curvature hollow core fiber in extremely high material loss region," Opt. Exp., vol. 21, no. 8, pp. 9514-9519, 2013.

[7] N. V. Wheeler et al.."Low Loss and Low Bend Sensitivity MidIR Guidance in a Hollow Core-Photonic Bandgap Fiber," Opt. Lett., vol. 39, no. 2, 2014.

[8] A. Urich et al., "Delivery of high energy Er:YAG pulsed laser light at 2.94um through a silica hollow core photonic crystal fibre," Opt. Express, vol. 20, no. 6, pp. 9160-9167, 2012.

[9] N. K. Baddela et al. "First Demonstration of a Low Loss 37-cell Hollow Core Photonic Bandgap Fiber and its Use for Data Transmission," in CLEO/QELS, 2013, p. CTu2K.3.

[10] L. S. Rothman et al. "The HITRAN 2008 molecular spectroscopic database," J. Quant. Spectrosc. Radiat. Transf., vol. 110, no. 9-10, pp. 533-572, 2009. 